\title{
Therapeutic Prospects of MicroRNA in Human Cancer
}

\section{Xiongbin $\mathrm{Lu}^{*}$ and Guohui Wan}

Department of Cancer Biology, The University of Texas MD Anderson Cancer Center, Houston, Texas, USA

Advances in genetics and molecular biology have improved our knowledge of the inner functions of cells, the basic building blocks of the body. Behaviors of each human cell are controlled by its collection of genetic information, which is included in about 25,000 genes in humans. These genes and their products, RNAs and proteins, instruct the cell what to do and when to grow and divide. According to the central dogma of molecular biology, RNAs, transcribed from genes on the chromosomal DNA, are in charge of transferring genetic information and carry out DNA instructions by producing proteins that guide each aspect of cell activities. However, this central dogma is getting challenged by the recent findings that a family of small nonprotein-coding RNAs, typically $\sim 22$ nucleotides in length, namely microRNAs (miRNAs) are able to inhibit protein-coding genes by interfering with the stability and function of mRNAs. Accumulating studies have highlighted critical roles of miRNAs as central posttranscriptional repressors of gene expression [1]

\section{miRNAs - A Family of Small Non-Coding RNAs}

MiRNAs are small endogenous noncoding RNAs that regulate gene expression by repressing translation or promoting the degradation of their target mRNAs. It has become an emerging concept that miRNAs could interact with cellular networks to exert their function. They are involved in almost every biological process, including development, differentiation, cell-cycle control, apoptosis, and cancer. MiRNAs regulate gene expression by binding to the $3^{\prime}$ untranslated region (UTR) of their target mRNAs and mediating mRNA degradation or translational inhibition. In the human genome, approximately transcripts of $30 \%$ to $50 \%$ of genes are estimated to be targeted by miRNAs [2,3].

MiRNAs are produced through sequential endonucleolytic cleavage mediated by two evolutionarily conserved RNase III enzymes, Drosha and Dicer [4]. They can be transcribed from two different pathways. Approximately half of miRNAs (intergenic miRNAs) are derived from non-coding RNA transcripts, whereas intronic or exonic miRNAs are located within protein-coding genes and share a common promoter with their host genes. The majority of miRNAs is transcribed by RNA Pol II as primary miRNAs (pri-miRNAs). Following transcription, the pri-miRNA is recognized and processed by the Drosha-DGCR8 microprocessor in the nucleus. This first cleavage step generates an approximately 70-nucleotide hairpin structure called precursor miRNA (premiRNA), containing 25 to 30 base pair stems and relatively small loops with 3 ' overhangs. Next, the pre-miRNA is exported from the nucleus through an interaction with exportin-5, a RanGTP-binding nuclear transporter. The pre-miRNA is then further processed by Dicer, generating a 20 to 25 nucleotide double-stranded mature miRNA consisting of a functional guide strand and passenger strand. Dicer associates with Ago2 and TRBP/PACT to form the RNA-induced silencing complex (RISC), loading complex (RLC), allowing the tight coupling of Dicer cleavage to the incorporation of miRNA into the RISC complex [5]. While the passenger strand is degraded, the mature miRNA is loaded into the RNA-induced silencing complex (RISC) where it directs Ago2 to target mRNAs and repress protein expression.

\section{miRNAs as Tumor Suppressors and Oncogenesm}

When mammalian cells exhibit aberrant activities such as abnormal growth and loss of apoptosis, they become precancerous and eventually result in cancer initiation. A number of studies have shown that miRNA regulates cell growth and apoptosis [6]. As an example, miR-15 and miR-16 induce apoptosis by targeting antiapoptotic gene BCL2 mRNA, a crucial player in many types of human cancers, including leukemias, lymphomas, and carcinomas [7]. Utilizing many molecular techniques, which include quantitative PCR, miRNA microarray, altered expression of miRNAs, and RNAome deep sequencing, many miRNAs have been identified as key players in human cancers, including lung, breast, brain, liver, colon cancer, and leukemia. Proof-of-principle studies validate a number of miRNAs as oncogenes or tumor suppressors. More than $50 \%$ of miRNA genes are located in cancer-associated genomic regions or in fragile sites, suggesting that miRNAs may play a more important role in tumorigenesis than previously thought [8]. Overexpressed miRNAs in cancers, such as mir-17-92, may function as oncogenes and promote tumor progression by inhibiting tumor suppressor genes that control cell differentiation or apoptosis [9]. Silenced miRNAs in cancers may function as tumor suppressor genes and may inhibit cancers by downregulating oncogenes. miRNA expression profiles have become useful biomarkers for cancer classification and diagnostics. It is predicted that miRNA-based therapy will be a powerful tool for cancer prevention and treatment.

The initial evidence demonstrating the involvement of miRNAs in human cancers came from a pioneering study on miR-15a/miR16-1 in human chronic lymphocytic leukemia (CLL). Calin et al. first showed that these two miRNAs are located on a chromosomal region that is frequently deleted in 50\% of B-cell CLL cases [7]. Further studies identified anti-apoptotic gene BCL2 as one of the primary target of miR-15a/miR-16-1. Meanwhile, the Slack group identified oncogene RAS as specific targets for miRNAs in the let-7 family [10]. In silico prediction was first used to identify genes with 3' UTRs (untranslated regions) containing let-7 complementary sites. The top candidate targets are the nematode RAS gene and the human KRAS, HRAS, and NRAS genes. Supporting this finding is the fact that genomic regions commonly deleted in lung cancer (where RAS is a cancer-initiating oncogene) contain several human let-7 genes. RNA expression profiles revealed specific downregulation of let-7 expression and concomitant overexpression of RAS in samples of lung, as compared with normal adjacent tissue. In addition to tumor suppressor miRNAs, miRNAs with oncogenic properties can negatively regulate tumor suppressor

*Corresponding author: Xiongbin Lu, Department of Cancer Biology, The University of Texas MD Anderson Cancer Center, USA, Tel: 714-745-6247; Fax: 713-792-8747; E-mail: xlu2@mdanderson.org

Received July 04, 2012; Accepted July 04, 2012; Published July 06, 2012

Citation: Lu X, Wan G (2012) Therapeutic Prospects of MicroRNA in Human Cancer. J Integr Oncol 1:e102. doi:10.4172/2329-6771.1000e102

Copyright: (c) 2012 Lu X. This is an open-access article distributed under the terms of the Creative Commons Attribution License, which permits unrestricted use, distribution, and reproduction in any medium, provided the original author and source are credited. 
proteins. One of the oncogenic miRNAs (oncomiRs) is miR-21. miR21 was reported to be aberrantly overexpressed in breast tumors, glioblastoma and pancreatic cancer [11]. In fact miR-21 targets the tumor suppressor's phosphatase and tensin homolog (PTEN) and programmed cell death 4 (PCDC4). In the past decade, more than a dozen of miRNAs have been shown to play important roles in tumor initiation, progression and metastasis. More recently, miRNAs have been reported to impact response to cancer drugs in chemotherapy and targeted therapies. For examples, overexpression of miR-221 and miR-222 is responsible for resistance to anti-estrogen therapies $[12,13]$. Inhibition of miR-21 and miR-200b increases sensitivity to gemcitabine [14], a nucleoside analog used in various carcinomas: non-small cell lung cancer, pancreatic cancer, bladder cancer and breast cancer.

\section{miRNAs in Cancer Prognosis and Treatment}

Deregulated miRNA expression in human cancer is due to chromosomal abnormalities (amplification, deletion and translocation), mutations, polymorphisms (SNPs), deficient miRNA biogenesis machinery and epigenetic changes of miRNA genes as well [15]. Genome-wide profiling showed that miRNA expression signatures allowed various types of cancer to be discriminated with high accuracy and the origin of poorly differentiated tumors to be verified. In one study, Rosenfeld et al. established a classifier based on 48 miRNAs from miRNA microarray analysis of 253 samples [16,17]. Two-thirds of samples were classified with high confidence, with accuracy $>90 \%$. Classification accuracy reached $100 \%$ for most tissue classes, including 131 metastatic samples. This finding demonstrates the effectiveness of miRNAs as biomarkers for identifying the tissue of origin for cancers of unknown primary origin. Recent progress in developing miRNA biomarkers allows us to detect miRNAs in blood and other human fluids. It has been shown that miRNAs circulate in a form of microvescicles called exosomes. Therefore, they are extremely stable and resistant to degradation. Weber et al. determined miRNA expression in 12 different types of body fluids (amniotic fluid, breast milk, bronchial lavage, cerebrospinal fluid, colostrum, peritoneal fluid, plasma, pleural fluid, saliva, seminal fluid, tears and urine) collected from healthy individuals, and showed that the highest concentrations of miRNAs were found in tears [18]. The ability to detect miRNAs in body fluids has generated great interest in their clinical potential as cancer biomarkers. Many studies have demonstrated that miRNAs can indeed be successfully employed both as cancer diagnostic and prognostic biomarkers both in solid and in hematological malignancies.

The ability to target multiple genes or biological processes makes miRNAs one of the most promising agents for cancer therapy. Initial evidence of the feasibility and efficacy of a miRNA-based therapy came from preclinical models aimed to understand the biological roles of a specific miRNA. As examples, delivery of miR-15a and miR16 into human prostate cancer cells induces apoptosis and inhibits tumor growth in vivo in a xenograft model [19]. Inhibiting miR-21 by antisense oligonucleotides exerts an apoptotic response in vitro in different types of cancer cells and also reduces tumor development and metastasis in vivo [11]. Despite the fact that basic knowledge of miRNA biology is still providing new insights, the relative ease by which miRNA can be manipulated pharmacologically appears to provide an intriguing opportunity for the treatment of disease. To date, there are several tools available to selectively target miRNA pathways. One method is to increase miRNA levels by delivering chemically synthesize miRNA mimics in vivo. miRNA mimics are synthetic RNA duplexes designed to mimic the endogenous functions of miRNA with chemical modifications for stability and cellular uptake. Synthetic miR- 34a was recently shown to inhibit lung tumor growth in a mouse model $[20,21]$. To target oncogenic miRNAs, the most widely used approach is to generate antimiRs that are modified antisense oligonucleotides harboring the full or partial complementary reverse sequence of a mature miRNA of interest. Stability, specificity and binding affinity are the key requirements for an antimiR to achieve its efficacy in vivo. Two types of chemical modifications have been used for this purpose, including 2'-O-methyl-group modification and locked nucleic acid (LNA)-modification.

Similar to other therapeutic oligonucleotides, the main challenge is the successful delivery of the therapeutic miRNAs to the target tissue without compromising the integrity of the miRNA. Development of clinical miRNA formulations often involves a complete evaluation of existing technologies to identify those that are amenable to the miRNA and its chemistry. Criteria in the evaluation process include sufficient delivery to induce a therapeutic effect in tumor models and a significant safety margin at therapeutic levels. Several delivery methods have proven effective in delivering therapeutic miRNAs to tumor tissues in vivo. These include viral vector-based systems and nanoparticle-based systems. In recent years, a variety of nanoparticles have been designed and developed. For example, systemic delivery of a miR-16 mimic inhibited metastasis of PC-3M prostate cancer cells intra-cordially injected 4 days before treatment [19]. The therapeutic delivery was facilitated using atelocollagen, a cationic polymer that associates with RNA through electrostatic interactions and forms particles in the nanometer diameter range. Because atelocollagen is a natural product, these nanoparticles are highly biocompatible and are able to achieve tumor-specific delivery via enhanced permeability and retention. Another development of miRNAs in cancer therapeutics is miR-34a. Therapeutic delivery of a miR-34a mimic using a neutral lipid emulsion, either by direct injections into the tumor or by systemic tail vein injections, prevented the outgrowth of viable subcutaneous lung tumor xenografts [20]. Importantly, miR-34a mimics formulated in the neutral lipid emulsion failed to induce elevated levels of neither cytokines nor liver and kidney enzymes in serum, suggesting that tumor inhibition was a specific effect of the mimic and that treatment was well tolerated. While recent progresses provide the experimental bases for the utilization of miRNAs as both targets and tools in cancer therapy, two major issues remain be addressed, which are development of genetically engineered animal models to study cancer-related miRNAs at various steps of tumorigenesis, and further improvement of miRNA mimics and antagomiRs delivery under clinical contexts.

\section{Concluding Remarks}

The past decade has witnessed an explosion of research focused on miRNAs. Accumulating evidence reveals that miRNAs play key roles in regulating tumor initiation, progression and metastasis. While cancer is a very complex disease, we are still at the verge of understanding miRNAs in various types of cancer. Nevertheless, our current knowledge of miRNAs has nourished the emergence of a wide spectrum of alternative translational applications that involve miRNAs and their associated molecules and pathways, including tumor classification, diagnosis, prognosis and prediction of overall survival for cancer patients, and administration of effective therapeutic targeting using miRNA mimics and antagomiRs. Combinations of miRNAs with current cancer therapeutics are believed to be highly effective towards improving the well-being of cancer patients. 


\section{References}

1. Siomi H, Siomi MC (2010) Posttranscriptional regulation of microRNA biogenesis in animals. Mol Cell 38: 323-332.

2. Winter J, Jung S, Keller S, Gregory RI, Diederichs S (2009) Many roads to maturity: microRNA biogenesis pathways and their regulation. Nat Cell Biol 11: $228-234$

3. Zhang X, Lu X (2011) Posttranscriptional regulation of miRNAs in the DNA damage response. RNA Biol 8: 960-963.

4. Bartel DP (2009) MicroRNAs: target recognition and regulatory functions. Cell 136: 215-233.

5. Davis BN, Hata A (2009) Regulation of MicroRNA Biogenesis: A miRiad of mechanisms. Cell Commun Signal 7: 18

6. Croce CM (2009) Causes and consequences of microRNA dysregulation in cancer. Nat Rev Genet 10: 704-714.

7. Cimmino A, Calin GA, Fabbri M, lorio MV, Ferracin M, et al. (2005) miR-15 and miR-16 induce apoptosis by targeting BCL2. Proc Natl Acad Sci U S A102: 13944-13949.

8. George AC, Carlo MC (2006) MicroRNA signatures in human cancers. Nat Rev Cancer 6: 857-866.

9. Aqeilan RI, Calin GA, Croce CM (2010) miR-15a and miR-16-1 in cancer: discovery, function and future perspectives. Cell Death Differ 17: 215-220.

10. Johnson SM, Grosshans H, Shingara J, Byrom M, Jarvis R, et al. (2005) RAS is regulated by the let-7 microRNA family. Cell 120: 635-647.

11. Krichevsky AM, Gabriely G (2009) miR-21: a small multi-faceted RNA. J Cell Mol Med 13: 39-53.
12. Miller TE, Ghoshal K, Ramaswamy B, Roy S, Datta J, et al. (2008) MicroRNA-221/222 confers tamoxifen resistance in breast cancer by targeting p27Kip1. J Biol Chem 283: 29897-29903.

13. Zhao JJ, Lin J, Yang $H$, Kong W, He L, et al. (2008) MicroRNA-221/222 negatively regulates estrogen receptor alpha and is associated with tamoxifen resistance in breast cancer. J Biol Chem 283: 31079-31086.

14. Meng F, Henson R, Wehbe-Janek H, Ghoshal K, Jacob ST, et al. (2007) MicroRNA-21 regulates expression of the PTEN tumor suppressor gene in human hepatocellular cancer. Gastroenterology 133: 647-658.

15. Iorio MV, Ferracin M, Liu CG, Veronese A, Spizzo R et al. (2005) MicroRNA gene expression deregulation in human breast cancer. Cancer Res 65: 70657070

16. Geva-Zatorsky N, Rosenfeld N, Itzkovitz S, Milo R, Sigal A, et al. (2006) Oscillations and variability in the p53 system. Mol Syst Biol 2: 2006.0033.

17. Rosenfeld N, Aharonov R, Meiri E, Rosenwald S, Spector Y, et al. (2008) MicroRNAs accurately identify cancer tissue origin. Nat Biotechnol 26: 462-469.

18. Weber JA, Baxter DH, Zhang S, Huang DY, Huang KH, et al. (2010) The microRNA spectrum in 12 body fluids. Clin Chem 56: 1733-1741.

19. Bonci D, Coppola V, Musumeci M, Addario A, Giuffrida R, et al. (2008) The miR-15a-miR-16-1 cluster controls prostate cancer by targeting multiple oncogenic activities. Nat Med 14: 1271-1277.

20. Chen Y, Zhu X, Zhang X, Liu B, Huang L (2010) Nanoparticles modified with tumor-targeting scFv deliver siRNA and miRNA for cancer therapy. Mol Ther 18: $1650-1656$

21. Wiggins JF, Ruffino L, Kelnar K, Omotola M, Patrawala L, et al. (2010) Development of a lung cancer therapeutic based on the tumor suppressor microRNA-34. Cancer Res 70: 5923-5930. 